\title{
Subdiagnóstico de restricción de crecimiento fetal mediante la aplicación de las curvas de crecimiento intrauterino del Ministerio de Salud
}

\author{
Jorge A Carvajal ${ }^{1,2}$, Claudio Vera P-G 1-3, Paula Vargas I, \\ Felipe Jordán U ${ }^{1}$, Alejandro Patillo G ${ }^{1}$, Enrique 0 yarzún $E^{1,2}$. \\ Under diagnosis of fetal growth \\ restriction by the new growth curves \\ of the Chilean Ministry of Health
}

\begin{abstract}
Background: Fetal growth restriction (FGR) is associated with increased risk of perinatal morbidity or death. Nationwide implementation of new fetal growth charts, requires a lower fetal weight for the diagnosis of FGR, compared to previous ones. This may lead to an under diagnosis of FGR in a large proportion of neonates. Aim: To compare the morbidity, mortality and anthropometry of neonates with FGR, diagnosed by MINSAL and Juez curves, with normal weight newborns in the same period (2000-2004). Material and methods: Revision of medical records of all births occurring in a maternity hospital between 2000 and 2004. The number of neonatal deaths, and the presence of hyperbilirubinemia, polyglobulia, hypoglycemia and hypothermia, were compared among children classified to be below percentile 10 of fetal growth according to both growth charts. Results: FGR was diagnosed in 4,4\% (502/11.289) and $9 \%(1.029 / 11.289)$ of newborns by MINSAL and Juez curves respectively. Compared to normal weight controls, the 527 newborns without FGR according to MINSAL curves, but below percentile 10 of Juez curves, had an odds ratio (OR) for polyglobulina of 8.14 (95\% confidence intervals (CI): 1.01-65.34), an OR for neonatal hypoglycemia of 5.10 (95\% CI: 1.11-23.39) and an OR for a ponderal index below $10^{\text {th }}$ percentile of 10.98 (95\% CI: 6.84-17.64). Conclusions: Newborns without a diagnosis of FGR by MINSAL curves but below $10^{\text {th }}$ percentile by Juez curves, have neonatal outcomes suggesting a true FGR. Juez curves should be maintained as a standard for the evaluation of fetal growth in our population (Rev Méd Chile 2007; 135: 436-42).
\end{abstract}

(Key words: Fetal development; Fetal viability; Perinatal care; Perinatal Mortality)

Recibido el 8 de junio, 2006. Aceptado el 3 de octubre, 2006.

Trabajo financiado por proyecto FONDECYT 1020675.

${ }^{1}$ Departamento de Obstetricia y Ginecología. ${ }^{2}$ Unidad de Medicina Matemo-Fetal. ${ }^{3}$ Unidad de Medicina Basada en Evidencia, Facultad de Medicina, Pontificia Universidad Católica de Chile.

Correspondencia a: Dr. Jorge A. Carvajal. Marcoleta 391, oficina 4. Centro de Investigaciones Médicas, Pontificia Universidad Católica de Chile. Santiago, Chile. Teléfono: 6863814. Fax: 6321924. E mail: jcarva@med.puc.cl 
L a restricción de crecimiento fetal (RCF) es a la imposibilidad de expresar el potencial genético de crecimiento del feto ${ }^{1}$. Los niños con RCF tienen un riesgo mayor de muerte o enfermedad (asfixia, hipoglicemia, hipocalcemia, hipotermia, poliglobulia e ictericia) en el período perinatal, y una mayor incidencia de parálisis cerebral, hipertensión arterial y diabetes en el seguimiento a largo plazo ${ }^{1}$.

No es posible conocer con certeza cuáles fetos no expresan su potencial genético de crecimiento, por lo que se ha establecido como definición operacional de restricción de crecimiento fetal al crecimiento bajo percentil 10 de la curva peso para la edad gestacional ${ }^{2}$. Esta definición operacional de RCF es universalmente aceptada, pues estos niños concentran un riesgo mayor de muerte 0 enfermedad ${ }^{3}$. Para el diagnóstico de RCF se requiere disponer de un patrón de crecimiento lo más ajustado posible a la población en control ${ }^{3}$.

En Chile, históricamente se utilizó como patrón de crecimiento, la curva de crecimiento fetal intrauterino de Lubchenco ${ }^{4}$. En 1984 fue reempla- zada por la curva de crecimiento intrauterino de Juez y cols ${ }^{5}$. Esta curva de crecimiento fue construida mediante el análisis prospectivo del peso de nacimiento de una cohorte de recién nacidos vivos únicos, sin malformaciones congénitas, hijos de madres sanas y sin factores de riesgo perinatal; nacidos en la maternidad de la Pontificia Universidad Católica de Chile, entre las 26 y 42 semanas de edad gestacional, entre los años 1978 y 1984.

En el año 2001, González y cols publicaron un nuevo patrón de crecimiento fetal ${ }^{6}$. En la elaboración de esta curva de crecimiento fetal, se consideró el peso de nacimiento de todos los recién nacidos únicos, vivos, nacidos en Chile entre los años 1993 y 2000, utilizando datos obtenidos del Instituto Nacional de Estadísticas (INE) y del Departamento de Informática del Ministerio de Salud de Chile. Esta curva de crecimiento fetal intrauterino fue adoptada por el Ministerio de Salud de Chile (MINSAL) desde el año 2004. En este nuevo patrón de crecimiento, el peso fetal en percentil 10 es menor que el de la curva de Juez y cols (Tabla 1).

Tabla 1. Percentil 10 de peso según edad gestacional para curvas Juez ${ }^{5}$ y M IN SAL ${ }^{6}$

\begin{tabular}{|cccc|}
\hline Edad gestacional & Juez (percentil 10) & MINSAL (percentil 10) & Diferencia (peso (gr)) \\
\hline 22 & - & 488 & - \\
23 & - & 511 & - \\
24 & - & 547 & - \\
25 & - & 595 & 199 \\
26 & 855 & 656 & 119 \\
27 & 850 & 731 & 85 \\
28 & 901 & 816 & 90 \\
29 & 1.001 & 911 & 116 \\
30 & 1.142 & 1.026 & 157 \\
31 & 1.316 & 1.159 & 202 \\
32 & 1.514 & 1.312 & 222 \\
33 & 1.730 & 1.508 & 219 \\
34 & 1.954 & 1.735 & 218 \\
35 & 2.178 & 1.960 & 202 \\
36 & 2.396 & 2.194 & 161 \\
37 & 2.598 & 2.437 & 139 \\
38 & 2.777 & 2.638 & 132 \\
39 & 2.924 & 2.792 & 109 \\
40 & 3.032 & 2.923 & 71 \\
41 & 3.093 & 3.022 & 14 \\
42 & 3.099 & 3.085 & \\
\hline
\end{tabular}

Se muestra el peso en gramos del percentil 10 de crecimiento para cada edad gestacional, según las curvas de crecimiento intrauterino de Juez ${ }^{5}$ y MINSAL ${ }^{6}$, y el cálculo de la diferencia en gramos entre ambos valores. 
Postulamos que la curva de crecimiento intrauterino MINSAL, publicada por González y cols ${ }^{6}$, subdiagnostica a los fetos con RCF. Para probar la hipótesis detectamos a aquellos recién nacidos ( $\mathrm{RN})$ no diagnosticados como RCF por la curva MINSAL, pero que sí correspondían a RCF según la curva de Juez en un período de 4 años en nuestra matemidad. Comparamos la morbilidad, mortalidad y antropometría de esos recién nacidos con un grupo control de crecimiento normal en el mismo período.

\section{MateRIAL Y MÉTOdo}

Se revisó la información de todos los recién nacidos en la Maternidad del Hospital Clínico de la Pontificia Universidad Católica de Chile, entre enero de 2000 y diciembre de 2004, comespondiente a 11.586 niños. Se analizó el crecimiento fetal de todos estos niños mediante las curvas de crecimiento MINSAL ${ }^{6}$ y Juez ${ }^{5}$, seleccionando al grupo de recién nacidos cuyo peso cae bajo el percentil 10 según Juez, pero sobre el percentil 10 según MINSAL Este grupo de RN constituye el grupo estudio A. El grupo de RN que cae bajo el percentil 10 según la curva MINSAL, constituyó el grupo estudio B. Como grupo control se seleccionó, al azar, un grupo de RN con crecimiento entre los percentiles 25 y 75 según la curva de Juez. Los $\mathrm{RN}$ del grupo control fueron elegidos al azar de entre todos los $\mathrm{RN}$ con crecimiento en percentil 25-75, pareado por edad gestacional y sexo con el grupo estudio A.

En los tres grupos se evaluó la presencia de morbilidad neonatal caractenística de RCF: hiperbilimubinemia, poliglobulia, hipoglicemia e hipotemia. También se evaluó el número de muertes en el peńodo perinatal. Hiperbilimubinemia fue definida como un aumento en el nivel plasmático de bilimubina, que de acuerdo a la norma del servicio, requirió tratamiento con fototerapia. Poliglobulia fue diagnosticada ante el hallazgo de hematocrito $>65 \%$. Hipoglicemia fue definida como el hallazgo de glicemia (idealmente 2 valores) $<40 \mathrm{mg} / \mathrm{dl}$ en las primeras $72 \mathrm{~h}$ y $<45 \mathrm{mg} / \mathrm{dl}$ después de las $72 \mathrm{~h}$ de vida. Se consideró hipotermia al hallazgo de temperatura rectal menor de $36,5^{\circ} \mathrm{C}$, persistente, en las primeras $6 \mathrm{~h}$ de vida.

Para evaluar la antropometría neonatal, se calculó el índice ponderal (IP) o índice de Rohrer (IP =peso al nacer (gr) x 100/talla al nacer $(\mathrm{cm})^{3}$ ). Se evaluó el número de $\mathrm{RN}$ con IP bajo el percentil 10 para la edad gestacional, como un reflejo de $\mathrm{RN}$ enflaquecidos, que probablemente corresponden a verdaderos RCF.

En las comparaciones estadísticas se usó test de $\chi^{2}$. Se consideró una diferencia estadísticamente significativa cuando el valor $\mathrm{p}$ resultó $<0,05$.

\section{RESULTADOS}

En el peńodo estudiado, se registraron 11.586 recién nacidos. Se excluyeron del análisis $246 \mathrm{RN}$ por presentar información incompleta, y 51 por ser de edad gestacional $<26$ semanas (edad a la cual se inicia la curva de Juez). Se analizaron 11.289 recién nacidos, $1.029 \mathrm{RN}$ con crecimiento menor a percentil 10 según Juez (9\%) y 502 bajo percentil 10 según MINSAL $(4,4 \%)$. La diferencia entre ambos, $527 \mathrm{RN}(4,7 \%)$, constituye el grupo estudio A y los 502 diagnosticados por la curva MINSAL, el grupo estudio B (Tabla 2). El grupo control comesponde a una muestra de $527 \mathrm{RN}$ seleccionados al azar, pareados por edad gestacional y sexo, con el grupo estudio A, con crecimiento en percentil 25-75 según Juez (Tabla 2).

Morbilidad. La incidencia de hiperbilirrubinemia, no fue diferente entre los tres grupos estudiados (3,6\%; 4,1\%; 3,0\% grupo A, grupo B y control, respectivamente) (Tabla 3 y Figura 1). La incidencia de poliglobulia $(1,5 \% ; 1,8 \% ; 0,1 \%)$ y de hipoglicemia neonatal $(1,9 \% ; 3,7 \%$; $0,3 \%)$ fue similar entre ambos grupos estudio y significativamente mayor que el control $(p<0,05)$ (Tabla 3 y Figura 1$)$. La incidencia de hipotermia fue significativamente mayor en el grupo estudio B (3,4\%), comparado con el control $(0,1 \%)$ y el grupo estudio A $(0,7 \%)$.

La morbilidad neonatal combinada (cualquiera de las patologías anteriores) fue significativamente mayor en ambos grupos estudio, comparados con el control (3,4\%). Grupo estudio A (7,2\%; OR (IC 95\%): $2,02(1,13-3,61)$ vs control). Grupo B $(8,0 \%$; OR (IC 95\%): 2,27 $(1,13-4,06)$ vs control. No hubo diferencias entre los grupos estudio A y B.

Mortalidad. La mortalidad perinatal fue significativamente mayor en el grupo estudio B (39,3/1.000 RN vivos), comparado con el grupo estudio A (5,7/1.000 $\mathrm{RN}$ vivos) y el grupo control (1,9/1.000 RN vivos) (Tabla 4). La mortalidad perinatal no fue mayor en el grupo estudio A que en el control (Figura 1). El resultado del análisis estadístico no cambia si se 
Tabla 2. Selección de pacientes para grupo estudio y grupo control

\begin{tabular}{|lccc|}
\hline \multicolumn{2}{l}{ RN nacidos en el período evaluado } & 11.586 & \\
RN excluidos & 297 & $246(2 \%)$ & Información incompleta \\
& & $51(0,4 \%)$ & Menores a 26 semanas \\
RN incluidos & & 11.289 & \\
& Grupo Estudio B & Grupo Estudio A & Grupo Control \\
\hline \multirow{2}{*}{ pc 10 Juez } & $<$ pc 10 MINSAL & $<$ pc10 Juez & pc 25-75 Juez \\
$1.029(9 \%)$ & $502(4,3 \%)$ & $>$ pc 10 MINSAL & 527 \\
\hline
\end{tabular}

Recién nacidos en la maternidad de la Pontificia Universidad Católica de Chile (desde enero 2000 a diciembre de 2004). Se indica número de RN y en paréntesis el \% respecto del número de $\mathrm{RN}$ incluidos en el análisis.

Tabla 3. Incidencia de morbilidad neonatal atribuible a RCF

\begin{tabular}{|cccc|}
\hline & $\begin{array}{c}\text { Grupo Estudio A } \\
(524 \mathrm{RN} \text { vivos) }\end{array}$ & $\begin{array}{c}\text { Grupo Estudio B } \\
(483 \mathrm{RN} \text { vivos) }\end{array}$ & $\begin{array}{c}\text { Grupo Control } \\
\text { (526 RN vivos) }\end{array}$ \\
\hline $\begin{array}{c}\text { Hiperbilirrubinemia } \\
\text { OR (IC 95\%) }\end{array}$ & $19(3,6 \%)$ & $21(4,3 \%)$ & $16(3,0 \%)$ \\
Poliglobulia & $8(0,61-2,36)$ & $1,45(0,75-2,81)$ & \\
OR (IC 95\%) & $8,14(1,01-65,34)$ & $9(1,9 \%)^{*}$ & $1(0,2 \%)$ \\
Hipoglicemia & $10(1,9 \%)^{*}$ & $9,97(1,67-61,08)$ & $2(0,4 \%)$ \\
OR (IC 95\%) & $5,10(1,11-23,39)$ & $19(3,9 \%)^{*}$ & $10,73(2,76-41,67)$ \\
Hipotermia & $4(0,8 \%)$ & $17(3,5 \%)^{* *}$ & $1(0,2 \%)$ \\
OR (IC 95\%) & $4,04(0,45-36,27)$ & $19,15(3,24-113,40)$ & \\
\hline
\end{tabular}

Se muestra la incidencia de morbilidad neonatal atribuible a RCF en los grupos estudios y el grupo control. Se expresa como número de $\mathrm{RN}$ con el diagnóstico, y en paréntesis el porcentaje respecto del total de $\mathrm{RN}$ vivos en el grupo. Se indica OR e intervalo de confianza para $95 \%$ de cada grupo vs control. $*_{p}<0,05$ vs control; $*_{p}<0,05$ vs control y grupo estudio A.

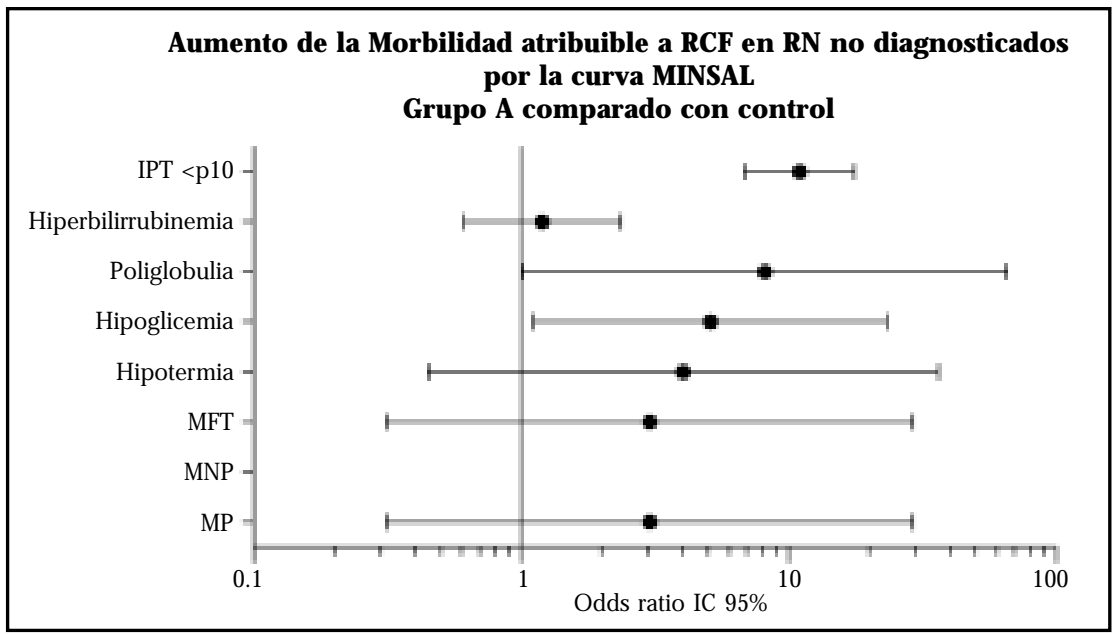

Figura 1. Se grafica Odds Ratio (OR) y el intervalo de confianza para el 95\%. En poliglobulia e hipoglicemia el intervalo de confianza no cruza el 1,0. MFT =mortalidad fetal tardía; $\mathrm{MNP}=$ mortalidad neonatal precoz; MP $=$ mortalidad perinatal. 
descartan aquellas muertes perinatales por causas no relacionadas con insuficiencia placentaria.

Índice ponderal. La incidencia de IP bajo percentil 10 fue similar en los grupos estudio A y B (31\% y $36 \%$, respectivamente), y significativamente mayor que en el grupo control (3,9\%) (Tabla 5 y Figura 1).

\section{DisCUSIÓN}

La utilización de la curva de crecimiento intrauterino de Juez, en nuestra población, clasifica a $9 \%$ de los RN como RCF, mientras la curva MINSAL coloca sólo a 4,3\% de los $\mathrm{RN}$ bajo el percentil 10. En el período estudiado, la curva MINSAL deja sin diagnóstico de RCF a 527 RN cuyo crecimiento es menor al percentil 10 de la curva de Juez, y diagnostica sólo a $502 \mathrm{RN}$ de los 1.029 identificados por Juez, es decir, omite casi la mitad de los diagnósticos.

La mortalidad neonatal fue significativamente mayor en los RN diagnosticados por la curva MNSAL, pero no en aquellos diagnosticados exclusivamente por Juez. Sin embargo, la morbilidad neonatal caractenística de RCF sí se encuentra significativamente incrementada en el grupo de RCF diagnosticado exclusivamente por Juez, es decir aquel grupo de RN cuyo diagnóstico se piende al hacer uso de la curva de crecimiento intrauterino MINSAL 6 .

Del mismo modo, observamos que el número de niños con índice ponderal (IP) menor al percentil 10 para la edad gestacional, es significativamente mayor en los RN con diagnóstico de RCF según Juez, pero no diagnosticados por la curva MINSAL, al compararlos con el grupo control.

La generación de patrones de crecimiento ajustados a las características de la población tratada, permitiendo un diagnóstico más certero de RCF, y así una mejor distribución de los recursos económicos y humanos, ha sido preocupación de múltiples grupos $^{2}$. La Organización Mundial de la Salud definió los criterios para que una curva de referencia sea considerada como un estándar apropiado ${ }^{7}$, sugiriendo que las curvas realizadas con grandes

Tabla 4. M ortalidad perinatal

\begin{tabular}{|lccc|}
\hline & Grupo Estudio A & Grupo Estudio B & Grupo Control \\
\hline Muerte fetal tardía & 3 & 15 & 1 \\
Muerte neonatal precoz & 0 & 4 & 0 \\
Mortalidad perinatal & $5,7 / 1.000$ & $39,3 / 1.000^{*}$ & $1,9 / 1.000$ \\
& $3,02(0,31-29,17)$ & $20,69(3,51-121,99)$ & \\
OR (IC 95\%) & & & \\
\hline
\end{tabular}

Se muestra el número de muertes en el período fetal tardío y neonatal precoz para cada grupo. Se indica mortalidad perinatal como número de muertes por $1.000 \mathrm{RN}$ vivos, se indica OR e intervalo de confianza para el 95\% vs grupo control. Las muertes fetales tardías comespondieron a fetos morfológicamente sanos, probablemente como producto de insuficiencia placentaria e hipoxia intrauterina. Las 4 muertes neonatales en el grupo estudio B se debieron a: 2 niños con trisomía 18; 1 niño con anencefalia y 1 niño con hemia diafragmática. $*_{p} \varangle 0,05$ vs control y grupo estudio A.

Tabla 5. Índice ponderal menor a percentil 10

\begin{tabular}{|cccc|}
\hline & $\begin{array}{c}\text { Grupo estudio A } \\
(527 \mathrm{RN})\end{array}$ & $\begin{array}{c}\text { Grupo estudio B } \\
(502 \mathrm{RN})\end{array}$ & $\begin{array}{c}\text { Grupo control } \\
(527 \mathrm{RN})\end{array}$ \\
\hline OR (IC 95\%) & $165(31,3 \%)^{*}$ & $181(36,1 \%)^{*}$ & $21(3,9 \%)$ \\
\hline
\end{tabular}

Se muestra el número de niños (y porcentaje entre paréntesis) con índice ponderal, al momento del nacimiento, menor al percentil 10 (según edad gestacional). Se indica OR e intervalo de confianza para $95 \%$ de cada grupo vs control.

IP =peso al nacer (gramos) x 100/talla al nacer $(\mathrm{cm})^{3}$.

$*_{p}<0,05$ vs control. 
muestras (como la de González y cols) serían más representativas de la población que aquellas curvas construidas en base a grupos seleccionados (como la de Juez y cols). La curva generada por González ${ }^{6}$, adoptada por el MINSAL, describe la población total de recién nacidos únicos vivos, nacidos en Chile entre 1993 y 2000, correspondiendo a un total de 2.049.446 niños, superando largamente los 200 niños en cada edad gestacional, y cumpliendo con todos los requerimientos indicados por la OMS.

Esta curva, sin embargo, no comesponde a un patrón de normalidad»s sino que a un patrón de «distribución poblacional»del peso al nacer. Aquellos $\mathrm{RN}$ hijos de madres con patologías que producen insuficiencia placentaria (hipertensión arterial crónica o inducida por el embarazo, diabetes pregestacional, etc), y así un menor crecimiento fetal que el esperado genéticamente, fueron incluidos en la muestra. Su inclusión disminuye el límite inferior de peso de nacimiento, lo que explica que el percentil 10 de crecimiento sea menor que el de la curva de Juez.

La curva propuesta por González y cols también incluyó a hijos de madres con patologías que determinan un crecimiento fetal mayor que el esperado genéticamente (diabetes gestacional, obesidad, aumento exagerado de peso en el embarazo), produciendo un aumento en el rango de peso superior. La inclusión de estos niños explica que en esta curva el percentil 90 sea mayor al percentil 90 de Juez.

Otro problema que estimamos serio respecto de la curva de González y cols, es el tipo de datos utilizados. Si bien los datos utilizados son de una fuente muy sólida (datos del Servicio de Registro Civil, compartidos por el Instituto Nacional de Estadísticas (INE) y el Departamento de Informática del Ministerio de Salud de Chile), nos parece que tienen un problema intrínseco al no corresponder a datos originados en registros médicos. La utilización del certificado de parto (que contiene la edad gestacional y el peso de nacimiento) no permite conocer si el cálculo de la edad gestacional fue el correcto y no permite conocer si el recién nacido o la madre eran portadores de una enfermedad.

Es importante, a nuestro modo de ver, comprender que esta curva de distribución poblacional no representa el crecimiento normal, pues hay una gran población no normal (no sana) que fue incluida en la elaboración de la curva. Sí nos interesa saber si un feto crece de modo normal (sano) y ajustado a su potencial genético de crecimiento, entonces requerimos de una curva de normalidad, como la de Juez.

La curva de Juez ${ }^{5}$ fue diseñada en una población seleccionada de fetos y madres sanos. Se siguió prospectivamente a una población de embarazadas sin factores de riesgo para crecimiento fetal exagerado o disminuido; con embarazos únicos, sin malformaciones congénitas, ni antecedentes de enfermedades maternas o fetales. Se incluyó a un total de $11.453 \mathrm{RN}$, entre las 26 y 42 semanas de edad gestacional, considerados sanos, y por tanto representativos de la normalidad. Es posible pensar que, con estas precauciones, los fetos incorporados fueron aquellos que expresaron correctamente su potencial de crecimiento. Nos parece que esta curva es mejor cuando se desea evaluar si un feto crece de modo normal o si cursa con una RCF.

La crítica a la curva de Juez es el reducido número de pacientes, especialmente en edades gestacionales menores (menos de 10 casos por semana antes de las 30 semanas). Creemos, sin embargo, que éste es un problema difícil de superar, pues es poco probable que un feto sano, hijo de una madre sana, tenga su parto a edad gestacional muy precoz. Si el parto se produce de modo prematuro, es muy probable que exista una patología, fetal o materna, que cause el parto prematuro; y es posible que este mismo factor etiológico cause un crecimiento fetal alterado.

En efecto, en una publicación previa reportamos que la incidencia de RCF entre los RN prematuros es el doble que la de los RN de término ${ }^{8}$. Postulamos que la isquemia útero-placentaria es el factor etiológico que relaciona el parto prematuro con la RCF. En nuestros estudios hemos aportado evidencia convincente para defender la hipótesis de la etiología isquémica para cerca de un tercio de los $\mathrm{RN}$ prematuros ${ }^{9}$. Es comprensible, entonces, que el peso neonatal de los RN prematuros se vea afectado por la causa del parto prematuro; y de modo poblacional es muy posible que el promedio de peso de los RN prematuros (y el nivel del percentil 10) disminuya por la inclusión de $\mathrm{RN}$ prematuros debido a isquemia útero-placentaria.

La OMS también ha reflexionado sobre este problema y en alguna medida coincide con nuestra posición. En su reporte ${ }^{7}$, se reconoce que el peso de nacimiento de los RN prematuros puede verse afectado por la causa del parto prematuro. Estiman, por ejemplo, que el peso de 
nacimiento de fetos provenientes de abortos (entre 20 y 24 semanas) es muy confiable, pero no así el de todos los $\mathrm{RN}$ de esa edad gestacional, si se considera a los partos espontáneos. Los expertos de la OMS concluyen, sin embargo, que es mejor usar curvas de referencia poblacional y no curvas normales, pese a reconocer lo anterior. Es probable que la recomendación de los expertos se haya visto influida por la necesidad política de concentrar recursos en aquellos fetos de altísimo riesgo y no en aquellos con riesgo de morbilidad menor.

Existen diferencias en los pesos estimados como normales por ambas curvas, diferencias que dejan a un grupo de fetos sin el diagnóstico de RCF según la curva MINSAL, pero que poseen el diagnóstico según la curva de Juez. Nuestro trabajo apunta a responder si este grupo de recién nacidos corresponde efectivamente a fetos con RCF o no. Es decir ¿se trata de un subdiagnóstico con la curva MINSAL o un sobrediagnóstico con la curva de Juez?

Evidenciamos que el grupo de recién nacidos no diagnosticados por la curva MINSAL, se comporta como verdaderos RCF, pues la incidencia de morbilidad atribuible a RCF (poliglobulia e hipoglicemia), así como el porcentaje de RN enflaquecidos (IP <pc10), es mayor que en la población control, y similar a la del grupo correctamente diagnosticado por ese patrón de crecimiento.

Concluimos que, en la población en control en nuestro hospital, el uso de las curvas de crecimiento

\section{REFERENCIAS}

1. Yanney M, Marlow N. Pediatric consequences of fetal growth restriction. Semin Fetal Neonatal Med 2004; 9: 411-8.

2. Gaziano EP. Antenatal ultrasound and fetal Doppler. Diagnosis and outcome in intrauterine growth retardation. Clin Perinatol 1995; 22: 111-40.

3. Clausson B, Gardosi J, Francis A, Cnattingius S. Perinatal outcome in SGA births defined by customized versus population-based birth weight standards. BJOG 2001; 108: 830-4.

4. Lubchenco LO, Hansman C, Dressler M, Boyd E. Intrauterine growth as estimated from live born birth weight data at 24 to 42 weeks of gestation. Pediatrics 1963; 32: 793-800.

5. Juez G, Ventura-JuncÁ P, Lucero E. Crecimiento intrauterino en un grupo seleccionado de recién de González y cols, actualmente incorporadas por el MINSAL, causa un subdiagnóstico de RCF. Las consecuencias de este subdiagnóstico son potencialmente graves, pues exponen a un grupo de niños con elevado riesgo de morbilidad, a un control prenatal estándar, similar al de la población normal, cuando lo correcto seńa efectuar un control especializado por un grupo de alto riesgo, y requiniendo la concentración de mayores recursos para disminuir la morbilidad de este grupo.

Podnía argumentarse que la curva MINSAL permite una mejor concentración de recursos en un grupo de alto riesgo de mortalidad perinatal. Estimamos, sin embargo, que la morbilidad a la que se exponen estos niños no diagnosticados es clínicamente significativa y no puede ser obviada. No evidenciamos una mayor mortalidad en el grupo con subdiagnóstico de RCF, hubo sólo una muerte fetal en el grupo control, y tres muertes fetales en este grupo subdiagnosticado (todas ellas atribuibles a insuficiencia placentaria). Si bien esta diferencia no fue estadísticamente significativa (5,7 vs $1,9 / 1.000 \mathrm{RN}$ vivos), marca una tendencia riesgosa si se proyecta a nivel poblacional.

En base a estos hallazgos, sugerimos mantener la utilización de la curva de crecimiento intrauterino de Juez y cols, como el patrón para analizar el crecimiento fetal, efectuando el diagnóstico de RCF a aquellos fetos bajo el percentil 10 de peso para la edad gestacional, y tratándolos como fetos de alto niesgo de morbilidad y mortalidad perinatal.

nacidos chilenos. Subdiagnóstico de Retardo de Crecimiento Intrauterino en Chile. Rev Méd Chile 1984; 112: 759-64.

6. Gonzáiez RP, Gómez RM, CASTro RS, Nien JK, Merino PO, ETCHEGARAY AB ET AL. Curva nacional de distribución de peso al nacer según edad gestacional: Chile, 1993 a 2000. Rev Méd Chile 2004; 132: 1155-65.

7. World Health Organization Expert Committee on the use and interpretation of anthropometry. Geneva, Switzerland: World Health Organization, 1995.

8. Carvajal JA, Morales Y, Germain AM. Restricción de crecimiento fetal: un hallazgo frecuente entre los recién nacidos prematuros. Rev Chil Obstet Ginecol 2001; 6: 463-71.

9. Germain AM, Carvajal Ja, Sánchez M, Valenzueia G, Tsunekawa H, Chuaqui B. Preterm labor: placental pathology and clinical correlation. Obstet Gynecol 1999; 94: 284-9. 